\title{
Spinocerebellar ataxia type 2 with glial cell cytoplasmic inclusions
}

\author{
S Probst-Cousin, T Acker, J T Epplen, M Bergmann, K H Plate, B Neundörfer, D Heuss
}

J Neurol Neurosurg Psychiatry 2004;75:503-505. doi: 10.1136/jnnp.2003.011825

Glial cell cytoplasmic inclusions were identified in a case of spinocerebellar ataxia type 2 . These have not been reported before. The inclusions were found in low frequency in the dentate nucleus, cerebellar white matter, pontine transverse fibres, and the inferior olivary nucleus. They were of variable size and shape and expressed ubiquitin, thus resembling glial cytoplasmic inclusions in multiple system atrophy. However, their immunohistochemical profile was different as they did not show immunoreactivity for either tau protein or $\alpha$-synuclein. There was no evidence of expanded polyglutamine tracts in these inclusions, which also failed to label with silver stains. As in many other neurodegenerative diseases, in spinocerebellar ataxia type 2 there may be pathogenic contributions of glial cells other than the common astrogliotic response to neuronal damage.

S pinocerebellar ataxia type 2 (SCA2) represents a genetically defined neurodegenerative disorder characterised by autosomal dominant inheritance and progressive cerebellar ataxia, combined with slow saccades and sensorimotor neuropathy. ${ }^{12}$ The neuropathology comprises olivo-ponto-cerebellar atrophy (OPCA) with axonopathy of posterior columns, spinocerebellar tracts, and peripheral nerves. ${ }^{3}$ Aetiologically, SCA2 is caused by a CAG trinucleotide repeat expansion in the coding region of the SCA2 gene on chromosome 12, leading to a polyglutamine tract in the encoded ataxin-2 protein, the function of which is still unclear. ${ }^{45}$ Recently, ubiquitinated neuronal intranuclear inclusions (NIs) of unclear pathogenic significance have been shown in some of the affected neuronal populations. ${ }^{6}$

Glial cell cytoplasmic inclusions have been described in different neurodegenerative diseases, including a case of SCA type $1 .^{78}$ These glial cell cytoplasmic inclusions differ according to distribution, immunological composition, and quantity. The only disorder described so far in which glial cell cytoplasmic inclusions represent the hallmark lesion is multiple system atrophy (MSA), a sporadic disease with a mixture of autonomic dysfunction, parkinsonism, cerebellar ataxia, and corticospinal symptoms. ${ }^{9-12}$

Here we describe ubiquitin positive glial cell cytoplasmic inclusions which we found haphazardly in the morphological examination of a case of SCA2, and we show that these inclusions are different from glial cell cytoplasmic inclusions in MSA.

\section{CASE REPORT}

At the age of 18 years, this male patient noticed impaired handwriting because of incoordination of his hands. Afterwards, he very slowly developed dysarthria and progressive walking and balance difficulties. At the age of 35 , physical examination showed a moderately severe dysarthria, slow saccades, and severe gait, trunk, and limb ataxia. There were also generalised fasciculations, distal loss of vibration sense, and generally absent deep tendon reflexes. Cranial tomography showed cerebellar and brain stem atrophy. At the age of 40, he was using a walker, and more severe limb ataxia was evident on finger-nose testing and rapidly alternating arm movements. He now also showed hypermetric saccades on lateral gaze, while his dysarthria became more mixed, with ataxic, spastic, and dystonic components. With exception of his lost vibration sense, sensory testing revealed no abnormalities. On the last examination at the age of 44, the patient was wheelchair-bound and he now also complained of frequency of urination, difficulty in emptying his bladder, and difficulty in swallowing. In the proximal muscles there was mild paresis and muscular atrophy. Because of his severe dysarthria, his speech was hardly intelligible; there were, however, no signs of cognitive impairment. Two months later he died of aspiration pneumonia.

A family history revealed a progressive ataxia affecting three generations, and genetic testing on our patient confirmed the diagnosis of SCA2 with 42 CAG repeats in the disease allele of the SCA2-gene. Unfortunately, genetic testing could not be carried out on other family members.

\section{METHODS}

Sections of brain and spinal cord were stained with haematoxylin and eosin (H\&E), cresyl violet, Luxol-fastblue, Bodian and Gallyas silver impregnations, and periodicacid-Schiff (PAS). Paraffin embedded and semi-thin sections of peripheral nerves (peroneal and sural) and muscles (gastrocnemius and quadriceps) were stained with toluidine blue, H\&E, and Gomori's trichrome.

Immunohistochemistry was done using the streptavidinbiotin complex/anti-alkaline phosphatase method, with fast red as chromogen. The primary antibodies applied were as follows: monoclonal mouse anti-human $\alpha$-synuclein (Transduction Laboratories, 1:250; Lexington , Kentucky, USA), monoclonal mouse-anti-human CD68 (Dako, 1:100; Glostrup, Denmark), monoclonal mouse anti-human tau protein AT8 (Innogenetics, 1:200; Ghent, Belgium), polyclonal rabbit anti-human ubiquitin (Dako, 1:100), monoclonal mouse anti-human glial fibrillary acid protein (GFAP) (Dako, 1:25), monoclonal mouse anti-human Leu7/CD57 (Becton Dickinson, undiluted; Oxford, UK), and monoclonal mouse anti-polyglutamine tracts IC2 (Chemicon, 1:100; Temecula, California, USA). For immunofluorescence staining, primary antibodies were coupled with Cy2 (carbocyanine, 1: 50; Dianova, Hamburg, Germany), excited by a light beam of $490 \mathrm{~nm}$, green signal at emission peak $519 \mathrm{~nm}$, or

Abbreviations: NI, neuronal intranuclear inclusion; MSA, multiple system atrophy; OPCA, olivo-ponto-cerebellar atrophy; SCA2, spinocerebellar ataxia type 2 
Cy3 (indocarbocyanine, 1: 50, Dianova), excited by a light beam of $550 \mathrm{~nm}$, red signal at emission peak $570 \mathrm{~nm}$; sections were observed under a reflected fluorescence microscope with mercury arc lamp (BX 51, Olympus, Hamburg, Germany). Double stained images were recorded and merged using image analysis software (analysis3.0, SIS, Münster, Germany). Two cases of MSA $^{13}$ served as controls.

\section{RESULTS}

Macroscopically, there was marked atrophy of both the pons and the cerebellum, whereas histologically, moderate neuronal loss and gliosis in the oculomotor nucleus and the substantia nigra was found. Most severely affected, however, was the basal pontine nucleus. We observed severe degeneration of transverse fibres and pontocerebellar fibre tracts, with distinct pallor of the white matter in Luxol-fast-blue myelin staining. In the medulla, marked degeneration of the inferior olives was seen. Both the cerebellar vermis and the cerebellar hemispheres showed moderate to marked loss of Purkinje cells with accompanying gliosis. The cerebellar white matter showed pallor in myelin staining, whereas in the dentate nucleus, only mild neuronal loss and gliosis were observed. In the spinal cord there was mild neuronal loss and gliosis of the anterior horn, with marked degeneration of the dorsal columns. The peripheral nerves showed severe reduction of large myelinated fibres, whereas in the muscles a neurogenic syndrome with angular atrophic fibres was observed.

GFAP staining highlighted astrogliosis in areas of degeneration, and CD68 positive macrophages were present in cerebellar and pontine white matter areas, commonly in the form of rod cells. Numerous ubiquitin positive non-specific dots and corpora amylacea were observed throughout the central nervous system. We found singular ubiquitin positive inclusions in the nuclei pontis and dentate nucleus in up to $3 \%$ of remaining neurones. In some Purkinje cell nuclei, diffuse positive staining for ubiquitin was seen. There was neuronal immunoreactivity for polyglutamine tracts as cytoplasmic granular deposits in Purkinje cells and singular neurones of the dentate nucleus. With decreasing frequency, there were ubiquitin reactive glial cell cytoplasmic inclusions in the dentate nucleus (up to four per high power field), in cerebellar white matter (up to three per high power field), in pontine transverse fibres (up to two per high power field), and in the inferior olivary nucleus (up to one per high power field). These inclusions were of variable size, their shape varying from triangular to sickle, oval, or flame shaped (fig 1A), thus resembling glial cell cytoplasmic inclusions in MSA. However, unlike the controls with MSA, they did not show immunoreactivity for either tau protein or $\alpha$-synuclein. There was co-localisation of glial cell cytoplasmic inclusions with Leu7/CD57 but not with GFAP or CD68, suggesting they were harboured in oligodendrocytes (fig 1B). The glial cell cytoplasmic inclusions were lacking immunoreactivity with the IC2 antibody and did not appear with any other stains such as H\&E, PAS, Luxol-fast-blue, cresyl violet, or the silver impregnations.

\section{DISCUSSION}

The presence of ubiquitinated cellular inclusions is a largely non-specific finding, merely indicating activation of cellular protein degradation. The significance of these inclusions is defined by the identity of the labelled protein. ${ }^{14}$ Most of the inclusion bodies in neurodegenerative diseases either contain tau protein or $\alpha$-synuclein, possibly because of their tendency to self associate and form pathological fibrils. ${ }^{15}$ As a key regulator of the cytoskeleton, tau protein is tightly associated with the microtubules whereas $\alpha$-synuclein is associated with synaptic termini and its primary function remains to be
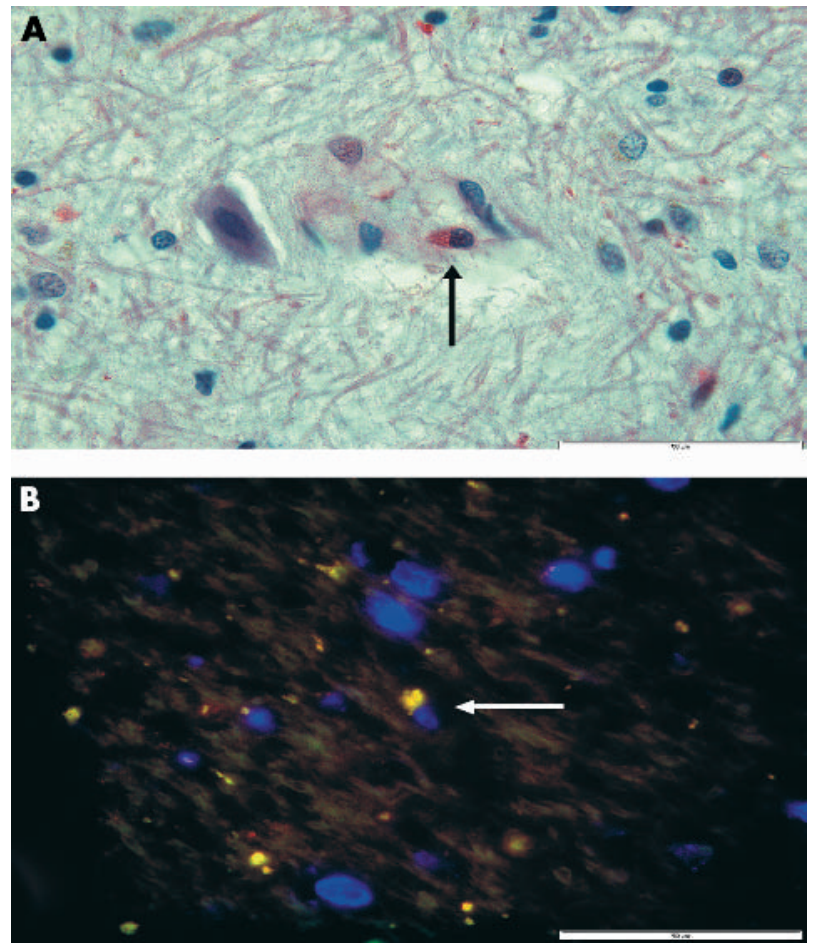

Figure 1 (A) Ubiquitin positive glial cytoplasmic inclusion in the vicinity of a neurone of the dentate nucleus (anti-ubiquitin immunohistochemistry, $\times 1000$, scale bar $=50 \mu \mathrm{m}$ ). (B) Merged image of immunofluorescence double labelling of ubiquitin (red Cy3 fluorescence) and Leu7/CD57 (green Cy2 fluorescence), co-localisation of ubiquitin and Leu7/CD57 indicated in yellow, $\times 1000$ magnification, scale bar $=50 \mu \mathrm{m}$.

determined. ${ }^{16}{ }^{17}$ The discovery of glial cell cytoplasmic inclusions in MSA has evoked considerable interest in glial pathology in various neurodegenerative diseases. ${ }^{7}{ }^{9}$ However, these glial inclusions are not uniform but differ according to cell type, distribution, and composition. Thus the descriptive term "glial cell cytoplasmic inclusions" was somewhat misleading as it covered very different lesions. Because of its nosological and pathognomonic impact, it currently refers in the strict sense to the oligodendroglial inclusions in MSA, whereas the glial changes in the other diseases appear to be either a manifestation of an underlying pathology, which involves both neurones and glial cells alike, or to represent a secondary glial reaction to primary neuronal lesions. ${ }^{79}$ The latter is also the most likely hypothesis for their presence in our case, as the lack of expression of expanded polyglutamine tracts, the restricted distribution in areas of neuronal degeneration, and the limited quantity of glial cell cytoplasmic inclusions suggest a secondary reactive or epiphenomenal role, without evidence for a significant contribution to white matter degeneration.

In hereditary ataxias, glial cell cytoplasmic inclusions have been described twice before. One case had hereditary OPCA with unclear genetic background, ${ }^{18}$ while the other had OPCA with the SCAl mutation presenting with glial cell cytoplasmic inclusions in an MSA-like distribution. ${ }^{8}$ The authors speculated on an overlap with MSA, concluding that the SCAl gene mutation may also result in a hereditary disorder similar to sporadic MSA. $^{8}$ However, a comprehensive study on possible genetic causes of MSA later found no evidence of the SCAl mutation underlying this condition. ${ }^{19}$ It would have been interesting to see if the glial cell cytoplasmic inclusions in that SCAl case expressed $\alpha$ synuclein. 
Though the morphological appearance is similar, the lack of immunoreactivity of glial cell cytoplasmic inclusions for both $\alpha$-synuclein and tau protein in our case discriminates them clearly from glial cell cytoplasmic inclusions in MSA, as $\alpha$-synuclein immunoreactivity, in particular, is relatively specific for MSA and Lewy body diseases. ${ }^{20}$ Glial cell cytoplasmic inclusions in other disorders-such as the filamentous oligodendroglial inclusions, called coiled bodies, of Alzheimer's disease, Pick's disease, dementia with argyrophilic grains, corticobasal degeneration, and progressive supranuclear palsy-usually lack $\alpha$-synuclein immunoreactivity. ${ }^{20-22}$ In contrast to the glial cell cytoplasmic inclusions described in this paper, coiled bodies share immunoreactivity for tau protein and show argyrophilia in Gallyas silver impregnation, though they usually fail to give a positive reaction to anti-ubiquitin antibodies. ${ }^{21}{ }^{22}$ Thus the immunohistochemical profile of the inclusions of our case is different from glial cell cytoplasmic inclusions and other common glial cell cytoplasmic inclusions.

It is not clear why glial cell cytoplasmic inclusions in SCA2 have not been described before. Possibly they might just have been overlooked as they are so few in number. Another reason could be that our SCA2 case had a special phenotype associated with the presence of glial inclusions. In this regard it could be speculated either that the SCA2 gene contains polymorphisms, or that additional modifying genes influence the phenotype. So far, we have only been able to investigate this single individual morphologically. Until our findings are reproduced, glial cell cytoplasmic inclusions in SCA2 will remain a non-specific finding of uncertain significance. The future will show whether SCA2 needs to be added to the growing list of diseases associated with glial pathology.

\section{ACKNOWLEDGEMENTS}

We are grateful to Ms C Winkelmann, Ms E Ganzmann, and Ms B Zieher for their excellent technical assistance. These investigations were supported in part by grant B26 of the IZKF University ErlangenNuremberg.

\section{Authors' affiliations \\ S Probst-Cousin, B Neundörfer, D Heuss, Department of Neurology, Friedrich-Alexander University, Erlangen, Germany \\ T Acker, K H Plate, Department of Neuropathology, Friedrich-Alexander University, Erlangen \\ J T Epplen, Department of Molecular Human Genetics, Ruhr University Bochum, Bochum, Germany \\ M Bergmann, Institute of Clinical Neuropathology, Central Hospital \\ Bremen-Ost, Bremen, Germany \\ Competing interests: none declared}

Correspondence to: Dr Stefan Probst-Cousin, Department of Neurology, Friedrich-Alexander University, Schwabachanlage 6, D-91054 Erlangen, Germany; stefan.probst-cousin@neuro.imed.uni-erlangen.de
Received 29 January 2003

In revised form 19 April 2003

Accepted 13 May 2003

\section{REFERENCES}

1 Bürk K, Abele M, Fetter M, et al. Autosomal dominant cerebellar ataxia type I. Clinical features and MRI in families with SCA1, SCA2 and SCA3. Brain 1996; 119:1497-505.

2 Filia A, De Michele G, Banfi S, et al. Has spinocerebellar ataxia type 2 a distinct phenotype? Genetic and clinical study of an Italian family. Neurology 1995;45:793-6.

3 Robitaille Y, Lopes-Cendes I, Becher M, et al. The neuropathology of CAG repeat diseases: review and update of genetic and molecular features. Brain Pathol 1997;7:901-26.

4 Gispert S, Twells R, Orozco G, et al. Chromosomal assignment of the second locus for autosomal dominant cerebellar ataxia (SCA 2) to chromosome 12q23-24.1. Nat Genet 1993;4:295-9.

5 Nechiporuk A, Lopes-Cendes I, Nechiporuk T, et al. Genetic mapping of the spinocerebellar ataxia type 2 gene on human chromosome 12. Neurology 1996:46:1731-5.

6 Koyano S, Uchihara T, Fujigasaki H, et al. Neuronal intranuclear inclusions in spinocerebellar ataxia type 2: triple labeling immunofluorescent study. Neurosci Lett 1999;273:117-20.

7 Chin SM, Goldman JE. Glial inclusions in CNS degenerative diseases. J Neuropathol Exp Neurol 1996;55:499-508.

8 Gilman S, Sima AAF, Junck L, et al. Spinocerebellar ataxia type 1 with multiple system degeneration and glial cytoplasmic inclusions. Ann Neurol 1996;39:241-55.

9 Lantos PL. The definition of multiple system atrophy: a review of recent developments. J Neuropathol Exp Neurol 1998;57:1099-111.

10 Papp MI, Kahn JE, Lantos PL. Glial cytoplasmic inclusions in the CNS of patients with multiple system atrophy (striatonigral degeneration, olivopontocerebellar atrophy and Shy-Drager syndrome). J Neurol Sci 1989;94:79-100.

11 Quinn NP. Multiple system atrophy-the nature of the beast. J Neurol Neurosurg Psychiatry 1989;52:78-89.

12 Wenning GK, Ben Shlomo Y, Magalhaes M, et al. Clinical features and natural history of multiple system atrophy. An analysis of 100 cases. Brain 1994; 117:835-45.

13 Probst-Cousin S, Bergmann M, Kuchelmeister K, et al. Ubiquitin-positive inclusions in different types of multiple system atrophy: distribution and specificity. Pathol Res Pract 1996;192:453-61.

14 Sherman MY, Goldberg AL. Cellular defenses against unfolded proteins: a cell biologist thinks about neurodegenerative diseases. Neuron 2001;29:15-32.

15 Dickson DW. Tau and synuclein and their role in neuropathology. Brain Pathol 1999;9:657-61.

16 Iwai A, Masliah E, Yoshimoto $M$, et al. The precursor protein of non-Ab component of Alzheimer's disease amyloid is a presynaptic protein of the central nervous system. Neuron 1995;14:467-75.

17 Kosik KS. The molecular and cellular biology of tau. Brain Pathol 1993;3:39-43.

18 Nakazato Y, Yamazaki H, Hirato J, et al. Oligodendroglial microtubular tangles in olivopontocerebellar atrophy. J Neuropathol Exp Neurol 1990;49:521-30.

19 Bandmann O, Sweeny MG, Daniel SE, et al. Multiple-system atrophy is genetically distinct from identified inherited causes of spinocerebellar degeneration. Neurology 1997;49:1598-604.

20 Wakabayashi K, Hayashi S, Kakita A, et al. Accumulation of alpha synuclein/NACP is a cytopathological feature common to Lewy body disease and multiple system atrophy. Acta Neuropathol 1998;96:445-52.

21 Lowe JS, Leigh N. Disorders of movement and system degenerations. In: Graham DI, Lantos PL, eds. Greenfield's Neuropathology, 7th ed. London: Arnold, 2002:325-430.

22 Mirra SS, Hyman BT. Ageing and dementia. In: Graham DI, Lantos PL, eds. Greenfield's Neuropathology, 7th ed. London: Arnold, 2002:195-271. 